\title{
Graphene-based nanovehicles for photodynamic medical therapy
}

This article was published in the following Dove Press journal:

International Journal of Nanomedicine

27 March 2015

Number of times this article has been viewed

Yan $\mathrm{Li}^{\prime}$

Haiqing Dong'
Yongyong Li'
Donglu Shi',2

'Shanghai East Hospital, The Institute for Biomedical Engineering and Nano Science (iNANO), Tongji University School of Medicine, Shanghai, People's Republic of China; ${ }^{2}$ The Materials Science and Engineering Program, Department of Mechanical and Materials Engineering, College of Engineering and Applied Science, University of Cincinnati, Cincinnati, $\mathrm{OH}$, USA
Correspondence: Donglu Shi

The Materials Science and Engineering Program, Department of Mechanical and Materials Engineering, College of Engineering and Applied Science, University of Cincinnati, 493 Rhodes Hall, ML72 University of Cincinnati, Cincinnati, USA

Tel + I 5 I35563I00

Email donglu.shi@uc.edu

Yongyong Li

Shanghai East Hospital, The Institute for Biomedical Engineering and Nano Science (iNANO), Tongji University School of Medicine, No. 155 Mailbox, Tongji University, 1239 Siping Road, Shanghai, 200092, People's Republic of China Tel +86 2l 65988029

Email yongyong_li@tongji.edu.cn
Abstract: Graphene and its derivatives such as graphene oxide (GO) have been widely explored as promising drug delivery vehicles for improved cancer treatment. In this review, we focus on their applications in photodynamic therapy. The large specific surface area of GO facilitates efficient loading of the photosensitizers and biological molecules via various surface functional groups. By incorporation of targeting ligands or activatable agents responsive to specific biological stimulations, smart nanovehicles are established, enabling tumor-triggering release or tumor-selective accumulation of photosensitizer for effective therapy with minimum side effects. Graphene-based nanosystems have been shown to improve the stability, bioavailability, and photodynamic efficiency of organic photosensitizer molecules. They have also been shown to behave as electron sinks for enhanced visible-light photodynamic activities. Owing to its intrinsic near infrared absorption properties, GO can be designed to combine both photodynamic and photothermal hyperthermia for optimum therapeutic efficiency. Critical issues and future aspects of photodynamic therapy research are addressed in this review.

Keywords: graphene, nanovehicle, photodynamic therapy, photosensitizer, hyperthermia

\section{Introduction}

Photodynamic therapy (PDT) has been extensively investigated for its high potential in medical treatment, especially in cancer therapy. ${ }^{1,2}$ As shown in Figure 1, the prominent feature of PDT, in comparison with other therapeutic means, is the utilization of light as an external trigger for selective activation of the photosensitizer (PS) within the targeted lesions. ${ }^{3}$ Typically, by directing a laser beam of a particular wavelength (for instance, $600 \mathrm{~nm}$ ) at the tumor cells and tissues with the internally uptaken photosensitizer, therapeutic species are released for effective killing of cancer cells. Upon light irradiation, the PS is activated to its excited triplet state via a short-lived singlet state. Subsequently, transfer of energy to the surrounding oxygen molecules can generate reactive oxygen species (ROS), such as singlet oxygen, superoxide anion radicals, hydroxyl radicals, and hydrogen peroxide. ROS are known for causing irreversible damage to tumor cells and tissues due to their highly cytotoxic effect (Figure 1). ${ }^{4}$

In PDT, PS is the key factor dominating the side effects and efficiency. The firstgeneration PSs were complex mixtures of several partially unidentified porphyrins. The limitations of porphyrins in clinical applications include poor selectivity, prolonged photosensitivity (low clearance rate), and low light penetration depth. ${ }^{5}$ In order to address these issues, second-generation PSs have been developed. These are the porphyrinoid derivatives (phthalocyanine, chlorine) and nonporphyrinoid PSs such as chalcogen-containing cationic dyes (eg, methylene blue [MB]), and the derivatives of hypocrellin, squaraine, and boron-dipyrromethene. ${ }^{6,7}$ The second-generation PS is an effective generator of singlet oxygen with strong absorption in the wavelength 


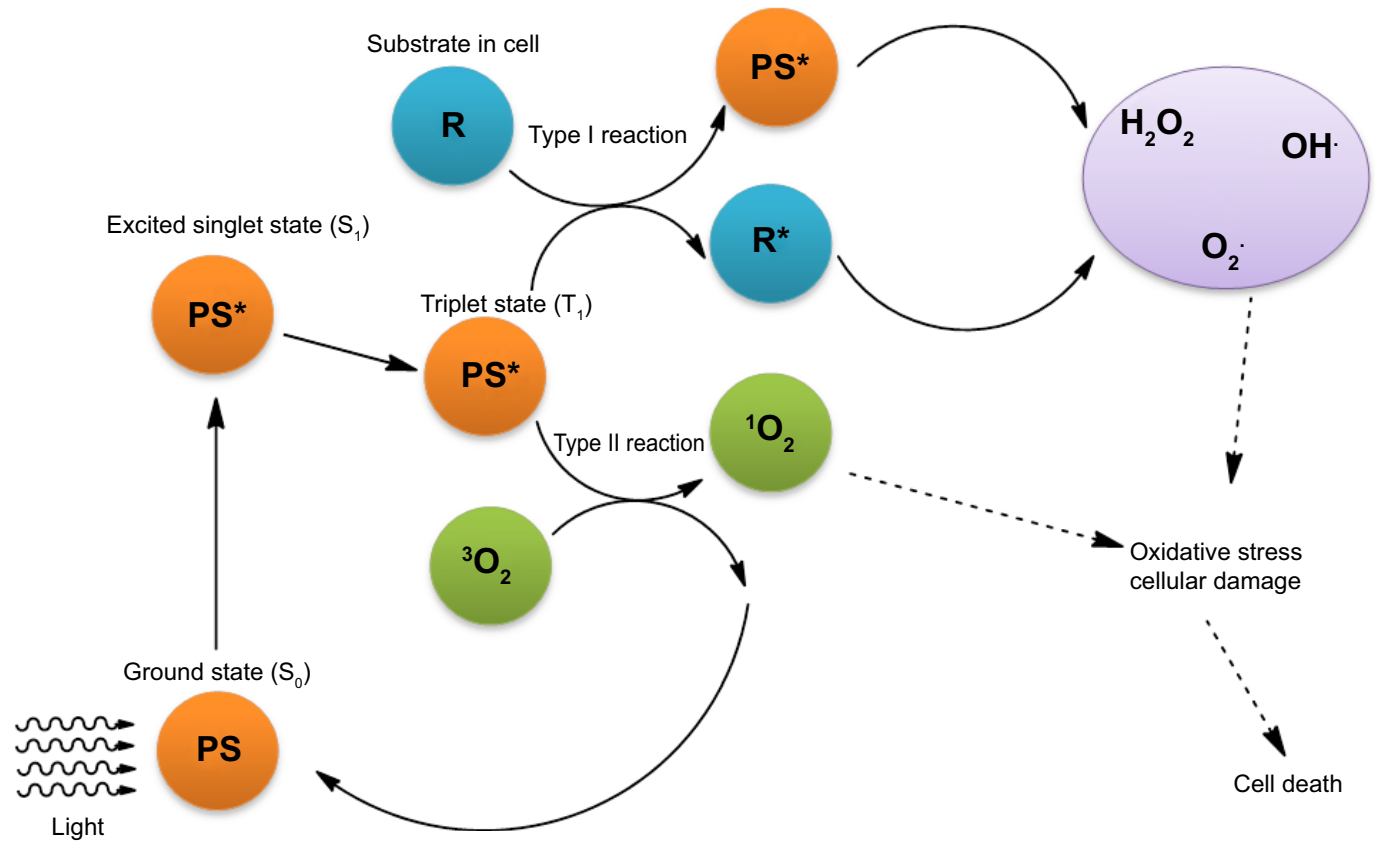

Figure I Schematic representation of PS-initiated cell death.

Notes: When PS in cells is exposed to a specific wavelength of light, PS in its singlet ground state $\left(\mathrm{S}_{0}\right)$ is transformed to its excited triplet state ( $\left.\mathrm{T}_{1}\right)$ via a short-lived excited singlet state $\left(\mathrm{S}_{1}\right)$ by intersystem crossing. The excited triplet can react directly either with substrate or solvent by transfer of hydrogen atom or electron to form radicals and radical ions upon interaction with oxygen. They can produce oxygenated products $\left(\mathrm{H}_{2} \mathrm{O}_{2}, \mathrm{O}_{2}^{--}\right.$, and $\left.\mathrm{OH}^{-}\right)$( Type I reaction) or can transfer their energy to oxygen directly to form singlet oxygen $\left({ }^{\prime} \mathrm{O}_{2}\right)$, which is a highly reactive, oxidative species (Type II reaction). Cellular damage is caused by these ROS, leading to tumor cell death. *Indicates activated state.

Abbreviations: PS, photosensitizer; ROS, reactive oxygen species.

range of 650-800 nm. However, most of these PSs are highly hydrophobic, easily subject to severe aggregation in aqueous medium. Their tumor selectivity is also poor. ${ }^{8}$ It is, therefore, important to develop advanced delivery systems and robust strategies that can address these critical issues. Several nanomaterials have been identified that have high aqueous solubility, bioavailability, and stability of hydrophobic PS. They also offer additional benefits of hydrophilicity and proper size for maximum tumor uptake via the enhanced permeability and retention effect. Furthermore, if designed properly, these nanomaterial systems can be assembled to carry active agents and targeting groups for enhanced tumorselective uptake and reduced side effects. ${ }^{8,9}$

Graphene and its derivatives such as graphene oxide (GO) offer ultrahigh surface areas with a single or a few layers of $\mathrm{sp}^{2}$ hybrid carbon atoms, making them ideal platforms for highly efficient drug loading. ${ }^{10-13}$ In particular, GO, a highly oxidized graphene with large numbers of residual carboxylic acid, hydroxide, and epoxide groups on its surfaces, can load various drug molecules via $\pi-\pi$ stacking, hydrophobic interactions, hydrogen bonding, and electrostatic interaction. Using various surface-coating strategies, including covalent and noncovalent conjugation, $\mathrm{GO}$ can be functionalized with versatile hydrophilic macromolecules to improve its biocompatibility and regulate its properties in biological systems. Furthermore, the GO surface can be easily modified with targeting ligands or active agents for selective or controlled drug delivery toward specific types of cancer cells. Owing to its intrinsic high optical absorption in the near-infrared (NIR) region, functionalized GO and reduced GO (RGO) have been utilized for photothermal ablation of tumors in animal experiments, with promising therapeutic efficiency. ${ }^{14-16}$

So far, considerable efforts have been devoted to exploring graphene-based nanomaterials in the PDT of cancer. In this review, we report the current progress in the study of PDT via nanotechnology. For different functional mechanisms of graphene in nanomaterial design, this review is mainly divided into two parts: 1) graphene designed as PS carrier; 2) graphene functionalized for acting as both PS carrier and photothermal agent. The essential issues concerning the further development of graphene-based nanomaterials in nanomedicine are addressed.

\section{GO as a PS carrier Organic PS delivery by GO}

The large $\pi$-conjugated structure of graphene allows for efficient loading of aromatic molecules and hydrophobic 
interactions. We previously reported ${ }^{17}$ loading of zinc phthalocyanine $(\mathrm{ZnPc})$ on the surface of methoxypoly(ethylene glycol)-conjugated nano-GO (NGO-mPEG). The drug-loading efficiency of NGO-mPEG has reached $14 \mathrm{wt} \%$, which is significantly higher than that of conventional carriers such as liposomes or micelles. ${ }^{18,19}$ Hydrophilic mPEG conjugation of NGO was found to enhance the solubility and stability of NGO in cell culture media. No apparent cytotoxicity of the NGO-mPEG was observed toward MCF-7 carcinoma cell line. NGO-mPEG, loaded with ZnPc, exhibited significant cytotoxicity toward cancer cells under Xe light irradiation (Figure 2).

GO can also be employed as a nanovehicle for loading different cargoes on its large surfaces (two accessible sides for single nanosheets). PEG-grafted GO (pGO) nanosheets were developed for co-delivery of the anticancer drug doxorubicin (Dox) and the photosensitizer chlorin e6 (Ce6) by physicochemical adsorption, resulting in combined chemophotodynamic therapy. ${ }^{20}$ The size of Ce6- and Dox-loaded pGO nanophysisorplexes (Ce6/Dox/pGO) was controlled at $148.0 \pm 18.0 \mathrm{~nm}$. In vitro and in vivo studies indicated significantly higher photodynamic anticancer effects upon co-delivery of Dox and Ce6 by pGO, compared to the delivery of Ce6 or Dox alone by the pGO nanosheets. PDT of mice tumor sections with Ce6/Dox/pGO showed substantial disruption of tumor nuclei, whereas the other groups did not.

In addition to $\pi-\pi$ stacking and hydrophobic interactions, the carboxyl, hydroxyl, and epoxide groups on the GO sheet can also facilitate hydrogen bonding with organic PS molecules such as hypocrellin A (HA) and hypocrellin B (HB) containing hydroxyl and carbonyl groups. Zhou et $\mathrm{al}^{21,22}$ reported loading of $\mathrm{HA}$ and $\mathrm{HB}$ onto $\mathrm{GO}$ with an average size of 200-400 $\mathrm{nm}$ through $\pi-\pi$ stacking and hydrogen bonding. Further study showed that incorporation of HA and HB into GO nanovehicles significantly improved the stability of HA and HB in contrast to that of free PS in aqueous solution, which is crucial for intravenous drugs. In vitro studies have demonstrated significant tumor cell damage by GO-HA and GO-HB upon light irradiation.

GO can also be used for delivery of positively charged organic PSs, such as MB, via electrostatic interaction because of its large number of carboxyl groups. ${ }^{23,24}$ Nanocomplexes formed via electrostatic interaction showed unique $\mathrm{pH}$ responsive properties. The PS release rate was accelerated under acidic conditions. The protonation of the carboxylates on GO and the interaction with MB molecules were found to be reduced after acid treatment.

\section{Inorganic PS delivery by GO}

On exposure to ultraviolet (UV) light, inorganic nanoparticles, such as $\mathrm{TiO}_{2}$ and $\mathrm{ZnO}$, can produce electrons and holes, leading to subsequent formation of ROS such as hydrogen peroxide, hydroxyl radical, and superoxide radicals. However, UV light cannot penetrate deeply into human tissues and thus is limited to superficial tumors. ${ }^{25,26}$ ROS produced by UV-mediated paths also have a short life span that is unable to provide a prolonged cancer-killing effect. ${ }^{26} \mathrm{Hu}$ et $\mathrm{al}^{27}$ found that $\mathrm{GO}$ on the surface of $\mathrm{TiO}_{2}$ can induce visible light-responsive activity, wherein $\mathrm{GO}$ acts as the electron sink for enhanced visible light photodynamic activities. GO was capable of binding to $\mathrm{TiO}_{2}$ through the functionalized carboxylic acid groups, which directly interact with the hydroxyl groups. Due to the high electrical conductivity of graphene,

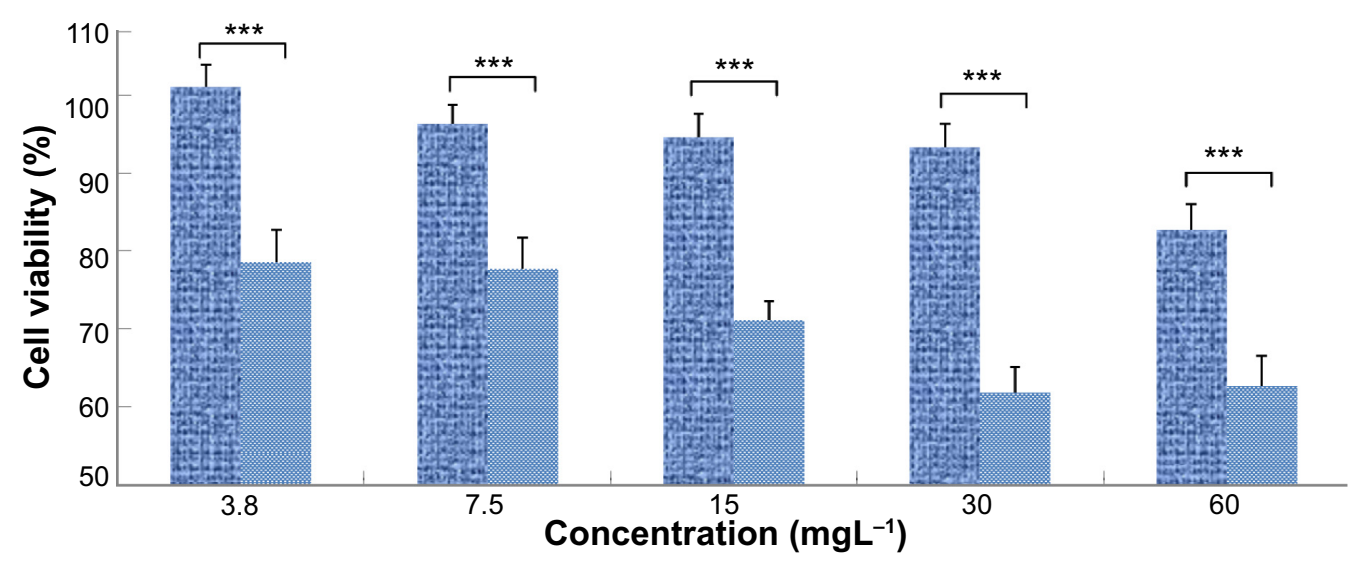

Figure 2 Cell viability assay at different concentrations of NGO-mPEG/ZnPc on MCF-7 cells.

Notes: The graph represents results obtained under 10-minute light exposure with power intensity of $60 \mathrm{~J} / \mathrm{cm}^{2}$ (light blue bars) and without light irradiation (dark blue bars). $* * * P<0.00$ I, Student's $t$-test.

Abbreviations: GO, graphene oxide; NGO-mPEG/ZnPc, methoxy-poly(ethylene glycol)-conjugated nano-GO loaded with zinc phthalocyanine. 
the photogenerated electron, which is produced by $\mathrm{TiO}_{2}$ under light, is gradually transferred from $\mathrm{TiO}_{2}$ to $\mathrm{GO}$, suppressing the electron-hole recombination effectively. According to this mechanism, holes in the valence band of $\mathrm{TiO}_{2}$ can migrate to the surface and react with $\mathrm{H}_{2} \mathrm{O}$ to form hydroxyl radicals $\left(\mathrm{OH}^{-}\right)$. The electron that arrives at $\mathrm{GO}$ reacts with oxygen to form $\mathrm{O}_{2}^{-}$or $\mathrm{O}_{2}^{-}$, which in turn combines with $\mathrm{H}^{+}$ to form hydrogen peroxide $\left(\mathrm{H}_{2} \mathrm{O}_{2}\right) . \mathrm{H}_{2} \mathrm{O}_{2}$ can react with $\mathrm{O}_{2}^{-}$. and reduce it to $\mathrm{OH}^{-}$. Thus, intracellular ROS accumulation can be efficiently increased by $\mathrm{GO} / \mathrm{TiO}_{2}$ hybrid for enhanced photodynamic activities. The photodynamic activity can cause lipid peroxidation and depolarization of mitochondrial membrane. It can also increase caspase-3 activity, inducing cell apoptosis and death (Figure 3).

$\mathrm{Hu}$ et al developed a targeting PS using folic acid (FA)functionalized $\mathrm{GO}$ containing $\mathrm{ZnO}$ nanoparticles on its surface. ${ }^{28}$ By hybridizing with GO-FA, the photocatalytic activities of $\mathrm{ZnO}$ nanoparticles were significantly enhanced, and the light absorption range was extended. Meanwhile, conjugation of the targeting agent FA onto the GO surface led to a remarkable improvement in tumor targeting, which was demonstrated by the cellular uptake assay. By generating ROS, GO-FA-ZnO caused antioxidant enzyme activity reduction and initiated various apoptotic events in HeLa cell lines.

\section{Targeted PS delivery by GO}

With targeting ligands, GO can enable targeted PS delivery to specific cells. In order to achieve more specific delivery of the PS molecule Ce6 to specific cells, Huang et al ${ }^{29}$ prepared a targeting drug delivery system in which GO (typically larger than $0.5 \mathrm{~mm}$ ) was conjugated with FA molecules (Figure 4). Ce6 was effectively loaded into the system with loading efficiency up to $\sim 80 \%$. The nanocarriers significantly increased the accumulation of $\mathrm{Ce} 6$ in tumor cells and led to a remarkable photodynamic efficacy on MGC803 cells upon irradiation.

Recently, switchable photo activity of PSs was reported based on a novel hyaluronic acid (HA)-GO conjugate system, with the size below $100 \mathrm{~nm}$ for targeted PDT. ${ }^{30}$ The conjugation of biocompatible and biodegradable HA onto GO sheets was found to increase the colloidal stability and biocompatibility of the GO nanosheets. It also specifically targeted the cancer cells with overexpressed HA receptors, thereby effectively improving the cellular uptake of PS. Loading efficiency was as high as $115 \%$ via $\pi-\pi$ stacking, as well as by hydrophobic interactions. The photo activity of $\mathrm{Ce} 6$ adsorbed on HA-GO nanocarriers was mostly quenched in aqueous solution to ensure biocompatibility, but this was quickly recovered after the release of $\mathrm{Ce} 6$ following cellular uptake. As a result, the PDT efficiency of the HA-GO/Ce6 nanohybrids was about ten-fold increased in comparison with that of free $\mathrm{Ce} 6$.

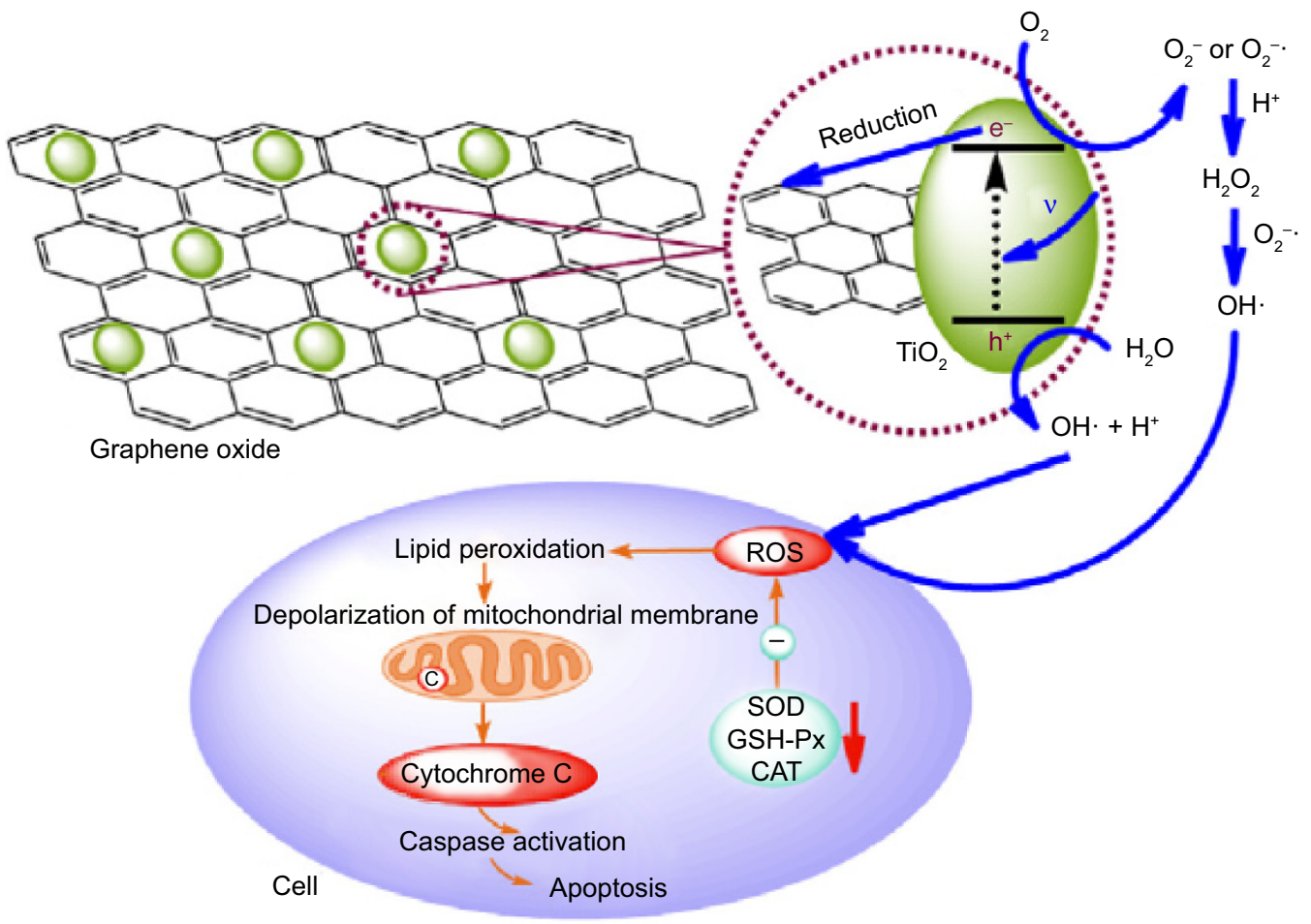

Figure 3 The hypothetical mechanism of synergistic enhancement in GOT and its photodynamic effects on cancer cells.

Notes: Reprinted from Carbon, 50, Zhen Hu, Yudong Huang, Shaofan Sun, Wenchao Guan, Yuhuan Yao, Peiyi Tang, Cuiyun Li, Visible light driven photodynamic anticancer activity of graphene oxide/ $\mathrm{TiO}_{2}$ hybrid, 994-1004, Copyright @2012, with permission from Elsevier. ${ }^{27}$

Abbreviations: CAT, catalase; GSH-Px, glutathione peroxidase; ROS, reactive oxygen species; SOD, superoxide dismutase; GOT, Graphene oxide/TiO 2 hybrid. 


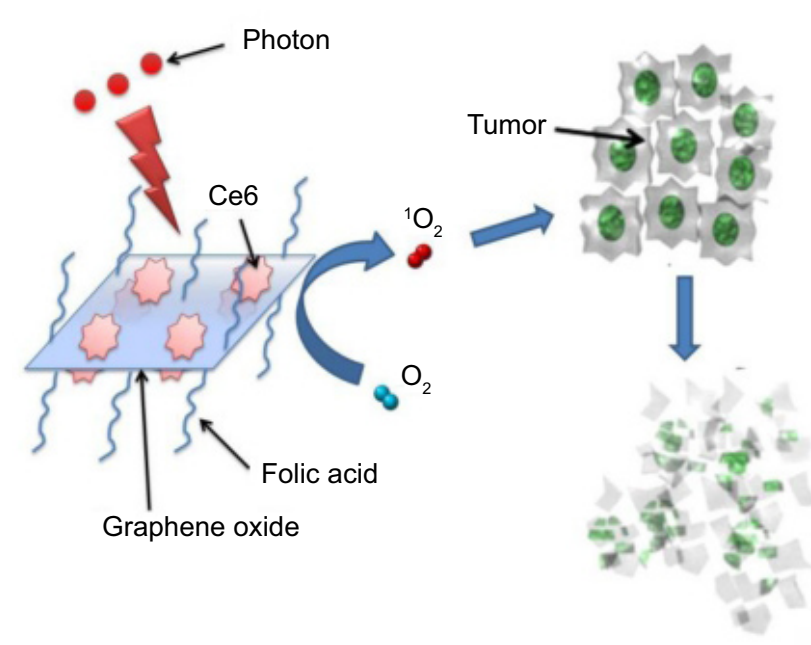

Figure 4 Photosensitizer molecules of Ce6 loaded by folic acid-conjugated graphene oxide.

Note: Reproduced with permission from Ivyspring International Publisher. Huang P, $\mathrm{Xu}$ C, Lin J, et al. Folic acid-conjugated graphene oxide loaded with photosensitizers for targeting photodynamic therapy. Theranostics. 20 I I; I:240-250. ${ }^{29}$ Abbreviation: Ce6, photosensitizer molecule Ce6.

\section{Target-activatable delivery of PS by GO}

Selective unpacking of PS at a specific site has shown great advantages for following tissue or cellular transfer and avoiding the quenching effect of GO carrier. GO-based drug delivery systems, responsive to environmental stimulations, have been developed for releasing PSs at a specific site.

Cho et $\mathrm{al}^{31}$ proposed a GO-Ce6 conjugate with a redoxresponsive cleavable disulfide linker (GO-SS-Ce6) as an activatable theranostic agent, in which the NIR fluorescence and the singlet oxygen generation (SOG) activities of $\mathrm{Ce} 6$ are activated by intracellular redox agents (in particular, glutathione [GSH]). GSH has been utilized as a molecular switch for triggered drug release, owing to its large concentration difference between the extracellular $(2 \mathrm{uM})$ and intracellular $(2-10 \mathrm{mM})$ compartments. GO-SS-Ce6 was nonfluorescent and nonphototoxic, even upon light irradiation. This is due to the fluorescence resonance energy transfer at the interfaces between GO and PSs at close proximity. Upon internalization of GO-SS-Ce6 into cells, disulfide bonds were rapidly cleaved in the presence of $\mathrm{GSH}$, resulting in the release of $\mathrm{Ce} 6$ from the GO complex and phototoxicity.

Following the above work, Cho et al ${ }^{32}$ developed a promising hyaluronidase (HAdase)-activatable GO-HA-Ce6 complex with overexpressed HAdase for various tumor cells. Noncovalent complex of GO and HA-Ce6 was prepared via 1) $\pi-\pi$ interaction between the GO planes and $\mathrm{Ce} 6$ molecules and 2) hydrophobic interaction between the GO planes and HA backbones. Due to energy transfer from the excited PSs to the GO surface in close proximity, the fluorescence intensity of HA-Ce6 was drastically quenched after loading of HA-Ce6 onto the GO surface. SOG from GO-HA-Ce6 was less than that of HA-Ce6 under the 670-nm laser irradiation. When exposed to HAdase, HA-Ce6 on the GO surface was degraded into small fragments, facilitating the release of $\mathrm{Ce} 6$ from the GO surface. As a result, the quenched Ce6 fluorescence was well recovered and the complex exhibited significant increases in SOG. In vitro cell viability assays indicated remarkably improved PDT efficacy with GO-HA-Ce6, compared to both free $\mathrm{Ce} 6$ and the HA-Ce6 conjugate. This improvement is associated with the highly enhanced intracellular uptake of GO-based carriers and the subsequent enzymatic activation of SOG by lysosomal HAdase.

\section{GO as both a PS carrier and photothermal agent Combination of PDT and photothermal therapy}

Apart from serving as drug carriers, graphene sheets can also act as photothermal agents for hyperthermic cancer therapy owing to their high optical absorption in the NIR region.

Tian et $\mathrm{al}^{33}$ reported that the PDT efficiency of nanographene can be further improved by a unique photothermal therapy (PTT). PEG-functionalized GO was loaded with the PS molecule Ce6 via $\pi-\pi$ stacking, yielding GO-PEG-Ce6 that generated singlet oxygen under laser irradiation for photodynamic cancer killing. It was found that GO-PEG-Ce6 exhibited a remarkable cancer cell photodynamic destruction effect compared to free $\mathrm{Ce} 6$, as a result of efficient cell entry of GO-PEG with Ce6. More importantly, they found that the photothermal effect of GO-PEG, under 808-nm NIR irradiation at a low power density, induced a mild local heating, which significantly increased cell uptake of GO-PEG-Ce6. The enhanced cell uptake was facilitated by high cell membrane permeability at a higher temperature. The synergistic photothermal and photodynamic effect further promoted cancer cell killing (Figure 5).

Sahu et $\mathrm{a}^{24}$ prepared a Pluronic noncovalently functionalized nano-GO, which was complexed with $\mathrm{MB}$ for combined PDT and PTT in in vivo cancer therapy. The nano-GO-MB complex efficiently delivered MB into cancer cells and showed an enhanced anticancer effect due to the combined PDT-PTT effect. Moreover, these nanoparticles showed high tumor accumulation when intravenously injected into the tumorbearing mice. The tumors were first irradiated with a $650-\mathrm{nm}$ laser for PDT using MB and were subsequently exposed to 

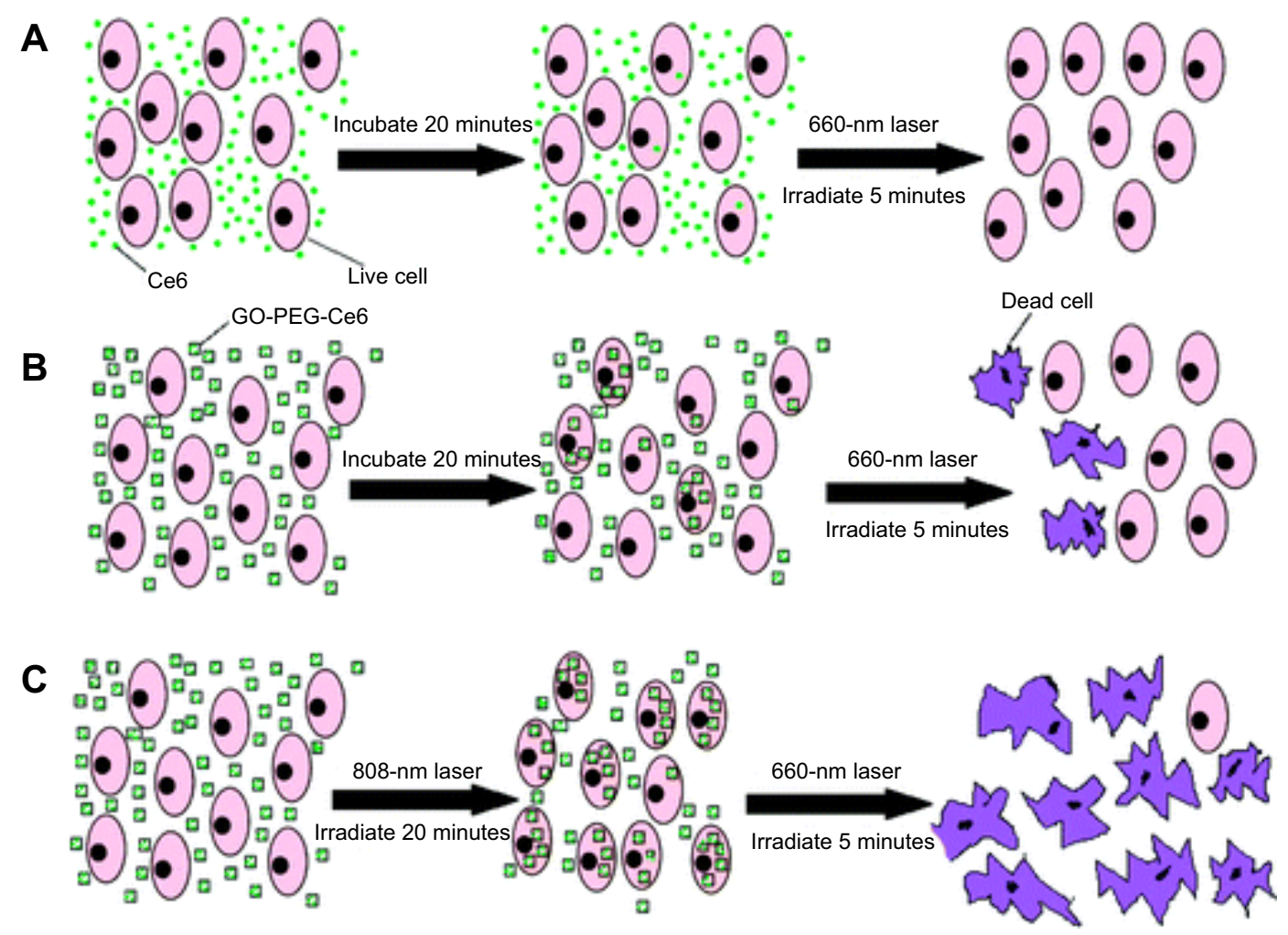

Figure 5 Schemes of the experimental design in photothermally enhanced photodynamic therapy.

Notes: KB cells were incubated with (A) free Ce6; (B) GO-PEG-Ce6 for 20 minutes in the dark; and then irradiated with $660-\mathrm{nm}^{2}$ laser $\left(50 \mathrm{~mW} / \mathrm{cm}^{2}, 5 \mathrm{minutes}\right.$, I5 J/cm $\left.{ }^{2}\right)$ in the control experiments, and (C) to induce the photothermal effect, cells incubated with GO-PEG-Ce6 were exposed to $808-\mathrm{nm}^{2}$ laser $\left(0.3 \mathrm{~W} / \mathrm{cm}^{2}, 20 \mathrm{minutes}, 360 \mathrm{~J} /\right.$ $\mathrm{cm}^{2}$ ) first before PDT treatment. Adapted with permission from Tian B, Wang C, Zhang S, Feng LZ, Liu Z. Photothermally enhanced photodynamic therapy delivered by nano-graphene oxide. ACS Nano. 201 1;5:7000-7009. Copyright @201 I American Chemical Society. ${ }^{33}$

Abbreviations: GO, graphene oxide; GO-PEG-Ce6, polyethylene glycol-functionalized GO photosensitizer molecule Ce6; PDT, photodynamic therapy.

an 808-nm laser that induced PTT by nano-GO. The in vivo results showed total ablation of tumor, indicating the pronounced synergistic effect of dual phototherapy (Figure 6).

Compared to GO, RGO exhibited higher intrinsic thermal conductivity and NIR absorbance. Therefore, RGO is favorable for applications in PTT. Chen et a ${ }^{34}$ reported a synergistic apoptotic therapy by a sequential irradiation process of highly integrated nanoassemblies based on the complexation of RGO-ZnO-HA (rGO-ZnO-HA). Compared with PDT or PTT alone, the combination of both resulted in a significant cytotoxicity. ROS generated by $\mathrm{ZnO} / \mathrm{RGO}$ under PDT can effectively sensitize cancer cells for subsequent NIR laserinduced apoptotic hyperthermia. This unique approach can effectively improve mild PTT (Figure 7). The surface engineering of $\mathrm{ZnO} /$ graphene hybrid with multifunctional $\mathrm{HA}$ biomacromolecules can tailor the system for optimum colloidal stability, biocompatibility, and cancer cell targeting.

\section{Image-guided combination of PDT and PTT}

As demonstrated by extensive previous experimental results, GO can be engineered to acquire highly integrated multiple functions in a single system. By conjugating with imaging probes, GO can be functionalized for biomedical diagnosis. Wang et $\mathrm{al}^{35}$ developed a theranostic nanocomposite for upconversion luminescence image-guided combinatorial PDT/PTT of cancer. The composite was synthesized by covalently grafting core-shell structured upconversion nanoparticles (UCNPs) with NGO through biofunctional PEG. ZnPc was then loaded onto the surface of NGO via $\pi-\pi$ stacking. The UCNPs-NGO/ZnPc nanocomposites were employed as the upconversion luminescence imaging probes in studies of cells and whole animals with high contrast for diagnosis. The system generated cytotoxic singlet oxygen under 630-nm laser irradiation for PDT. The UCNPs-NGO/ $\mathrm{ZnPc}$ nanocomposites also exhibited the photothermal effect that caused cancer cell killing (Figure 8). Compared with PTT or PDT alone, the combined treatment is shown to be a more efficient means of cancer therapy.

Recently, Gollavelli and Ling ${ }^{36}$ reported a single lightinduced photothermal and photodynamic reagent with dual-modal imaging capability. The theranostic reagent magnetic and fluorescent graphene (MFG)-SiNc4 [silicon napthalocyanine bis (trihexylsilyloxide)] was constructed by 


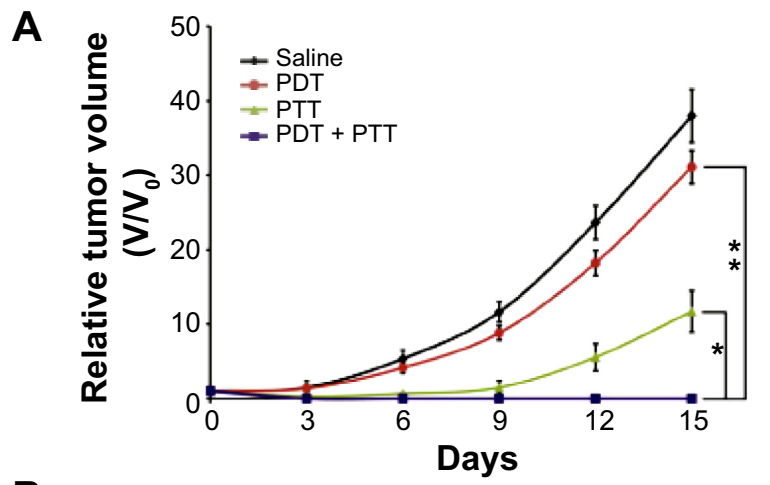

B

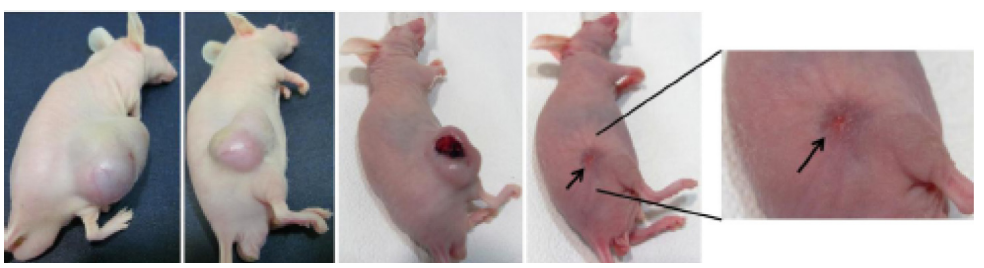

Figure 6 In vivo cancer therapy in HeLa tumor-bearing mice.

Notes: $(\mathbf{A})$ Changes in the relative tumor volume $(n=3)$ after different phototherapies. PDT only showed minimal effect on tumor growth, whereas PTT alone showed improved effect on tumor growth. The combination of PDT and PTT showed optimum therapeutic effect compared to PDT only $(* * P<0.00 \mathrm{I})$ or PTT only ( $* P<0.0 \mathrm{I})$. The dual therapy resulted in complete ablation of tumor tissue and no regrowth occurred within a span of I5 days. (B) Photographs of mice with tumors on the I5th day after treatment with saline, PDT only, PTT only, and PDT-PTT combined therapy. The mice with combined therapy showed no sign of tumor regrowth and the burned skin was also healed (the arrow indicates the healed site). P-values were calculated by the Student's $t$-test: $* P<0.0 \mathrm{I}$, $* * P<0.00 \mathrm{I}$. Reprinted from Biomaterials, 34 , Sahu A, Choi WI, Lee JH, Tae G, Graphene oxide mediated delivery of methylene blue for combined photodynamic and photothermal therapy, 6239-6248, Copyright @20I3, with permission from Elsevier. ${ }^{24}$

Abbreviations: PDT, photodynamic therapy; PTT, photothermal therapy.

noncovalent immobilization of the hydrophobic PS SiNc4 onto water-dispersible MFG via $\pi-\pi$ stacking. The MFG serves as an excellent luminescence image reagent and T2-weighted magnetic resonance imaging contrast reagent owing to its fluorescence and superparamagnetic properties. MFG-SiNc4 exhibits a broad NIR absorption (600-1,200 nm) that is effective for both PTT and PDT. Upon irradiation by NIR light, MFG-SiNc4 generates ${ }^{1} \mathrm{O}_{2}$-mediated photodynamic
A

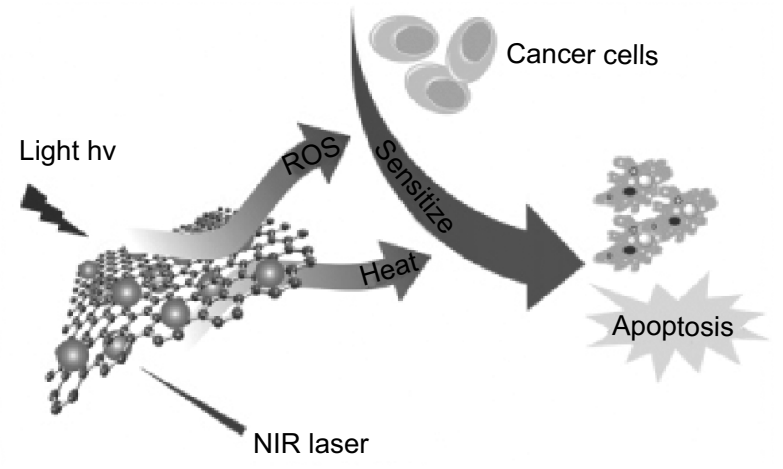

B

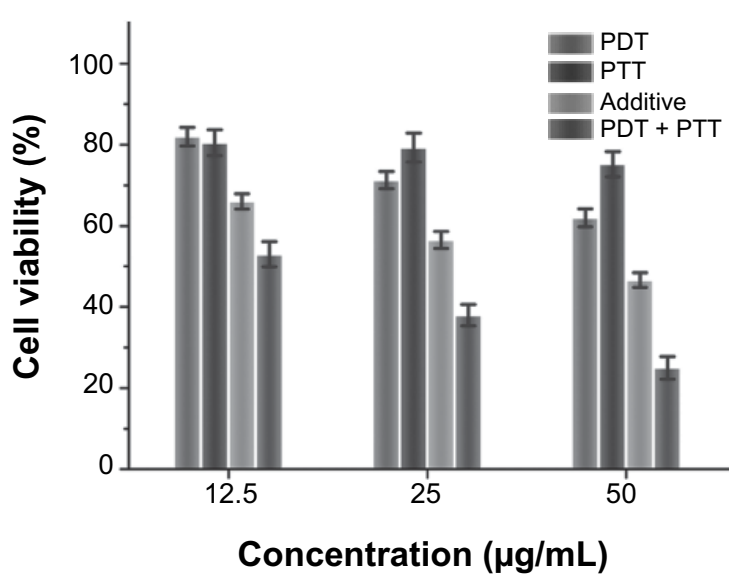

Figure 7 Irradiation-activated apoptosis.

Notes: (A) Schematic illustration of the sequential irradiation-activated high-performance apoptosis. (B) In vitro cell ability of MDA-MB-23I cells treated with rGO-ZnOHA following PDT, PTT, and combined PDT/PTT. The efficacy of combined treatment is compared with the additive efficacy of independent PDT and PTT treatments using $t$-tests with all $P$-values lower than 0.05. Reprinted from A multi-synergistic platform for sequential irradiation-activated high-performance apoptotic cancer therapy. Chen ZW, Li ZH, Wang JS, et al. Adv Funct Mater. 24:522-529. @20I3 WILEY-VCH Verlag GmbH \& Co. KGaA, Weinheim. ${ }^{34}$

Abbreviations: NIR, near infrared; PDT, photodynamic therapy; PTT, photothermal therapy; rGO-ZnO-HA, reduced graphene oxide-zinc oxide-hyaluronic acid complex; ROS, reactive oxygen species. 


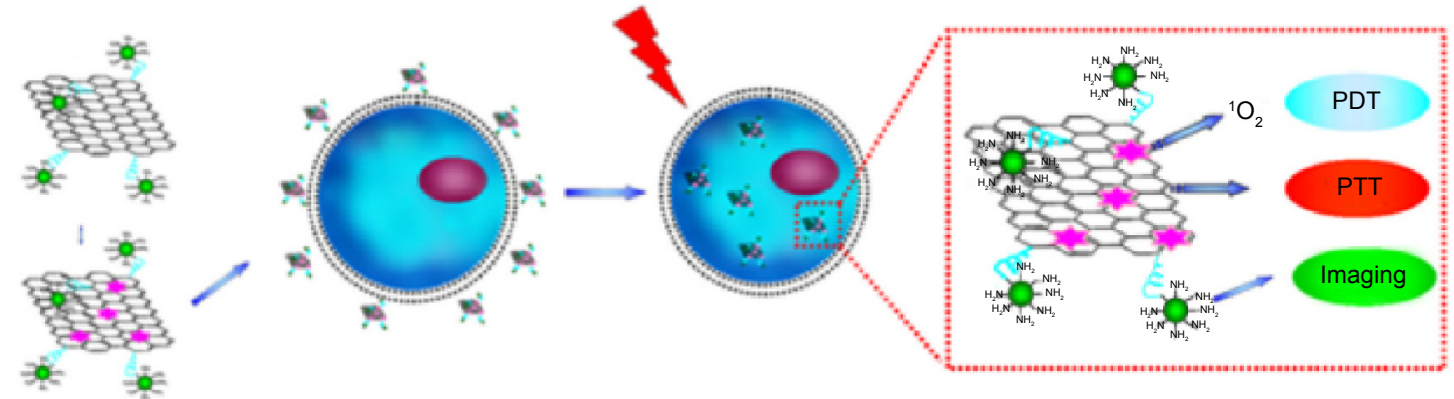

Figure 8 Schematic illustration of UCNP-NGO/ZnPc as a multifunctional theranostic nanoplatform for cancer treatment.

Notes: Reprinted from Biomaterials, 34, Wang YH, Wang HG, Liu DP, Song SY, Wang X, Zhang HJ. Graphene oxide covalently grafted upconversion nanoparticles for combined NIR mediated imaging and photothermal/photodynamic cancer therapy, 77I5-7724, Copyright @20I3, with permission from Elsevier. ${ }^{35}$

Abbreviations: GO, graphene oxide; PDT, photodynamic therapy; PTT, photothermal therapy; UCNP-NGO/ZnPc, upconversion nanoparticle-nano-GO-zinc phthalocyanine composite.

and photothermal effects. In vitro results have demonstrated simultaneous dual-modal imaging and PTT/PDT of MFGSiNc4, capable of cancer cell killing efficacy of $\sim 97.9 \%$. Of this, $\sim 64.7 \%$ was by PDT, whereas $\sim 33.2 \%$ was due to PTT. MFG-SiNc4 is therefore proven to be a potential theranostic reagent for dual-modal imaging-guided PDT/PTT cancer therapy with minimal side effects.

\section{Biosafety of graphene-based nanosystems}

Graphene-based nanosystems have shown great potential for PDT of cancer. However, biosafety of the nanomaterials must be taken into consideration. The toxicity and behavior of graphene-based materials in biological systems have been extensively investigated. ${ }^{37-42}$ Pristine graphene and GO have been found to be toxic to mice in a dose-dependent manner. But functionalized nano-GO (eg, by biocompatible surface coatings) is shown to be much less harmful in both in vitro and in vivo experiments. Surface modification of graphene has been found to effectively decrease its in vivo toxicity. Toxicity of graphene also depends on the chemical structure, charge, size, number of layers, and defects. Other factors include administration route, dose, time of exposure, as well as the cell types. Thus, more systematic investigations need to be carried out to fully understand the biological effects and to address safety concerns before implementation of clinical applications of any graphene-based materials.

\section{Conclusion and perspectives}

Graphene-based nanomaterials, mainly GO, have been extensively studied as an effective nanovehicle utilizing both organic PSs and inorganic nanoparticles such as $\mathrm{TiO}_{2}$ and $\mathrm{ZnO}$. Engineering of the nanovehicle relies on the large $\pi$-conjugated structure, abundant functional groups, and strong photoabsorbing properties in the NIR region. The unique physicochemical properties of graphene-based nanomaterials allow for efficient loading via both physical absorptions and chemical conjugations. Various strategies have been developed for GO-based PS delivery systems including targeted, target-activatable, and photothermally enhanced PDT. Upon incorporation of PS into the GO nanovehicles, the stability, bioavailability, and photodynamic anticancer effects of PSs can be significantly improved, with distinctive therapeutic effects. However, there are critical issues to be addressed before clinical applications. The primary concern is the external light absorption by the $\pi$-conjugated graphene, which interferes with the energy level transfer in PDT. In addition, these GO nanovehicles are generally in their pristine forms with highly dispersed particle sizes. More studies are required to find out the correlations between the physicochemical characteristics or structural modifications of graphenes and their biological impact. Rational, well-designed graphenes that can satisfy clinical requirements comprise the current challenges in the development of versatile GO-based nanocarriers for medical diagnosis and therapy.

\section{Acknowledgments}

This work was financially supported by 973 program (2013CB967500), the National Natural Science Foundation of China (51173136, 21104059 and 81402884), Shanghai Rising-Star Program (12QA1403400), and "Chen Guang" project founded by the Shanghai Municipal Education Commission and the Shanghai Education Development Foundation.

\section{Disclosure}

The authors report no conflicts of interest in this work. 


\section{References}

1. Wilson BC, Patterson MS. The physics, biophysics and technology of photodynamic therapy. Phys Med Biol. 2008;53:R61-R109.

2. Agostinis P, Berg K, Cengel KA, et al. Photodynamic therapy of cancer: an update. CA Cancer J Clin. 2011;61:250-281.

3. Brown SB, Brown EA, Walker I. The present and future role of photodynamic therapy in cancer treatment. Lancet Oncol. 2004;5:497-508.

4. Henderson BW, Dougherty TJ. How does photodynamic therapy work? Photochem Photobiol. 1992;55:145-157.

5. Dougherty TJ, Gomer CJ, Henderson BW, et al. Photodynamic therapy. J Natl Cancer Inst. 1998;90:889-905.

6. Detty MR, Gibson SL, Wagner SJ. Current clinical and preclinical photosensitizers for use in photodynamic therapy. J Med Chem. 2004;47: 3897-3915.

7. Yano S, Hirohara S, Obata M, et al. Current states and future views in photodynamic therapy. J Photochem Photobiol C Photochem Rev. 2011;12:46-67.

8. Chatterjee DK, Fong LS, Zhang Y. Nanoparticles in photodynamic therapy: an emerging paradigm. Adv Drug Deliv Rev. 2008;60:1627-1637.

9. Paszko E, Ehrhardt C, Senge MO, Kelleher DP, Reynolds JV. Nanodrug applications in photodynamic therapy. Photodiagnosis Photodyn Ther. 2011;8:14-29.

10. Liu JQ, Cui L, Losic D. Graphene and graphene oxide as new nanocarriers for drug delivery applications. Acta Biomater. 2013;9:9243-9257.

11. Yang K, Feng LZ, Shi XZ, Liu Z. Nano-graphene in biomedicine: theranostic applications. Chem Soc Rev. 2013;42:530-547.

12. Wang XM, Zhang WH. Application of graphene derivatives in cancer therapy: a review. Carbon N Y. 2014;67:795.

13. Dong HQ, Dong CY, Ren TB, Li YY, Shi DL. Surface-engineered graphene-based nanomaterials for drug delivery. J Biomed Nanotechnol. 2014;10:2086-2106.

14. Robinson JT, Tabakman SM, Liang Y, et al. Ultrasmall reduced graphene oxide with high near-infrared absorbance for photothermal therapy. J Am Chem Soc. 2011;133:6825-6831.

15. Yang K, Zhang S, Zhang G, Sun X, Lee S-T, Liu Z. Graphene in mice: ultrahigh in vivo tumor uptake and efficient photothermal therapy. Nano Lett. 2010;10:3318-3323.

16. Wang Y, Wang K, Zhao J, et al. Multifunctional mesoporous silicacoated graphene nanosheet used for chemo-photothermal synergistic targeted therapy of glioma. J Am Chem Soc. 2013;135:4799-4804.

17. Dong HQ, Zhao Z, Wen H, et al. Poly(ethylene glycol) conjugated nano-graphene oxide for photodynamic therapy. Sci China Chem. 2010;53:2265-2271.

18. Derycke AS, de Witte PA. Liposomes for photodynamic therapy. $A d v$ Drug Deliv Rev. 2004;56:17-30.

19. van Nostrum CF. Polymeric micelles to deliver photosensitizers for photodynamic therapy. Adv Drug Deliv Rev. 2004;56:9-16.

20. Miao W, Shim G, Lee S, Lee S, Choe YS, Oh YK. Safety and tumor tissue accumulation of pegylated graphene oxide nanosheets for codelivery of anticancer drug and photosensitizer. Biomaterials. 2013; 34:3402-3410.

21. Zhou L, Wang W, Tang J, Zhou JH, Jiang HJ, Shen J. Graphene oxide noncovalent photosensitizer and its anticancer activity in vitro. Chemistry. 2011;17:12084-12091.

22. Zhou L, Jiang HJ, Wei SH, Ge XF, Zhou JH, Shen J. High-efficiency loading of hypocrellin B on graphene oxide for photodynamic therapy. Carbon N Y. 2012;50:5594-5604.

International Journal of Nanomedicine

\section{Publish your work in this journal}

The International Journal of Nanomedicine is an international, peerreviewed journal focusing on the application of nanotechnology in diagnostics, therapeutics, and drug delivery systems throughout the biomedical field. This journal is indexed on PubMed Central, MedLine, CAS, SciSearch $\AA$, Current Contents ${ }^{\circledR} /$ Clinical Medicine,
23. Wojtoniszak M, Roginska D, Machalinski B, Drozdzik M, Mijowska E. Graphene oxide functionalized with methylene blue and its performance in singlet oxygen generation. Mater Res Bull. 2013;48:2636-2639.

24. Sahu A, Choi WI, Lee JH, Tae G. Graphene oxide mediated delivery of methylene blue for combined photodynamic and photothermal therapy. Biomaterials. 2013;34:6239-6248.

25. Cai R, Kubota Y, Shuin T, Sakai H, Hashimoto K, Fujishima A. Induction of cytotoxicity by photoexcited $\mathrm{TiO}_{2}$ particles. Cancer Res. 1992;52:2346-2348.

26. Blake DM, Maness P-C, Huang Z, Wolfrum EJ, Huang J, Jacoby WA. Application of the photocatalytic chemistry of titanium dioxide to disinfection and the killing of cancer cells. Sep Purif Method. 1999;28:1-50.

27. Hu Z, Huang Y, Sun S, et al. Visible light driven photodynamic anticancer activity of graphene oxide/ $\mathrm{TiO}_{2}$ hybrid. Carbon $N$ Y. 2012;50: 994-1004.

28. Hu Z, Li J, Li CY, et al. Folic acid-conjugated graphene-ZnO nanohybrid for targeting photodynamic therapy under visible light irradiation. J Mater Chem B. 2013;1:5003-5013.

29. Huang $\mathrm{P}, \mathrm{Xu} \mathrm{C}$, Lin J, et al. Folic acid-conjugated graphene oxide loaded with photosensitizers for targeting photodynamic therapy. Theranostics. 2011;1:240-250.

30. Li F, Park S-J, Ling D, et al. Hyaluronic acid-conjugated graphene oxide/photosensitizer nanohybrids for cancer targeted photodynamic therapy. J Mater Chem B. 2013;1:1678-1686.

31. Cho Y, Choi Y. Graphene oxide-photosensitizer conjugate as a redoxresponsive theranostic agent. Chem Commun. 2012;48:9912-9914.

32. Cho Y, Kim H, Choi Y. A graphene oxide-photosensitizer complex as an enzyme-activatable theranostic agent. Chem Commun. 2013;49: 1202-1204.

33. Tian B, Wang C, Zhang S, Feng LZ, Liu Z. Photothermally enhanced photodynamic therapy delivered by nano-graphene oxide. ACS Nano. 2011;5:7000-7009.

34. Chen ZW, Li ZH, Wang JS, et al. A multi-synergistic platform for sequential irradiation-activated high-performance apoptotic cancer therapy. Adv Funct Mater. 2014;24:522-529.

35. Wang YH, Wang HG, Liu DP, Song SY, Wang X, Zhang HJ. Graphene oxide covalently grafted upconversion nanoparticles for combined NIR mediated imaging and photothermal/photodynamic cancer therapy. Biomaterials. 2013;34:7715-7724.

36. Gollavelli G, Ling YC. Magnetic and fluorescent graphene for dual modal imaging and single light induced photothermal and photodynamic therapy of cancer cells. Biomaterials. 2014;35:4499-4507.

37. Bussy C, Ali-Boucetta H, Kostarelos K. Safety considerations for graphene: lessons learnt from carbon nanotubes. Acc Chem Res. 2013;46:692-701.

38. Yang K, Li YJ, Tan XF, Peng R, Liu Z. Behavior and toxicity of graphene and its functionalized derivatives in biological systems. Small. 2013;9:1492-1503.

39. Hu X, Zhou Q. Health and ecosystem risks of graphene. Chem Rev 2013;113:3815-3835.

40. Guo X, Mei N. Assessment of the toxic potential of graphene family nanomaterials. J Food Drug Anal. 2014;22:105-115.

41. Kostarelos K, Novoselov KS. Exploring the interface of graphene and biology. Science. 2014;344:261-263.

42. Seabra AB, Paula AJ, de Lima R, Alves OL, Duran N. Nanotoxicity of graphene and graphene oxide. Chem Res Toxicol. 2014;27:159-168.

\section{Dovepress}

Journal Citation Reports/Science Edition, EMBase, Scopus and the Elsevier Bibliographic databases. The manuscript management system is completely online and includes a very quick and fair peer-review system, which is all easy to use. Visit http://www.dovepress.com/ testimonials.php to read real quotes from published authors. 